\title{
Preparation and Photocurrent Properties of Tin Dioxide Electrode Modified by Polyimide Lagmuir-Blodgett Films Possessing Tetraphenylporphyrin Unit
}

\author{
Yasunari Nishikata, Atsushi Morikawa, Masa-aki KaKimoto ${ }^{\dagger}$ \\ Yoshio IMaI, Katsuhiko NishIYama,* \\ and Masamichi FUJIHIRA* \\ Department of Organic and Polymeric Materials and \\ * Department of Biomolecular Engineering, Tokyo Institute of Technology, \\ Meguro-ku, Tokyo 152, Japan
}

(Received December 18, 1989)

\begin{abstract}
Polyimide Langmuir-Blodgett (LB) film containing tetraphenylporphyrin (TPP) unit was newly prepared on the surface of tin dioxide electrode by "Precursor method" (Electrode I), and the generation of photocurrent was measured by the wet system. The photocurrent spectrum obtained was very similar in shape to the absorption spectrum of the LB film. The quantum efficiency of the electrode modified by one layer of the polyimide LB film was 0.178 , and the values decreased with increasing the number of layers. The long term stability of the photocurrent of Electrode I was compared with that of the electrode possessing the monomeric TPP LB film prepared from a 1:1 mixture of monoamino-TPP and stearic acid (Electrode II). These two electrodes were stable under the irradiation of monochromatic light of $430 \mathrm{~nm}$, while in the case of the direct irradiation of the xenon lamp, Electrode I kept the same level of the initial photocurrent for a long time, but Electrode II decreased to $50 \%$ within one hour. The absorption spectra indicated that both LB films did not come off from the surface of the electrodes during the photocurrent measurements. The decrease of the photocurrent of Electrode II was explained by the hydrophobicity of the surface of the LB film possessing the long alkyl chain and by the diffusing effect of the reducing agent in the electrolyte solution.

KEY WORDS Langmuir-Blodgett Film / Polyimide / Tin Dioxide / Tetraphenylprophyrin / Electron transfer / Photocurrent / Quantum Efficiency /
\end{abstract}

Porphines are well known as sensitizers working in the photosynthesis in plants, ${ }^{1}$ and in the artificial solar energy conversion systems. $^{2}$ Tetraphenylporphyrins (TPPs) are the typical synthetic model compounds in the porphine family. TPPs have been used for the modification of electrodes by vapor depositions, ${ }^{3-5}$ casting, ${ }^{4-6}$ and chemically bonding methods. ${ }^{7}$ One of the authors reported that the photocurrent was observed in the TPP modified electrode with high efficiency by irradiation of light, but the photocurrent became steeply down within a short period. ${ }^{7}$

Langmuir-Blodgett (LB) technique ${ }^{8}$ is one

$\dagger$ To whom all correspondence should be addressed. of the useful methods for the modification of electrode surface. ${ }^{9}$ Chlorophyll LB films prepared on the semiconductor electrodes were studied in connection with the mechanism of primary photosynthetic processes. ${ }^{10-12} \mathrm{We}$ have developed "Precursor method" for the preparation of polyimide LB films. ${ }^{13-15}$ The characteristics of the polyimide LB films were as follows: 1) Monolayer thickness of the LB films was about only one fourth of usual fatty acid LB films ${ }^{16}$ because of the absence of the long alkyl chain. 2) The polyimide LB films had high stability against water and organic chemicals, as well as high temperature stability. 
3) The polyimide LB films had much less amount of defects compared with the fatty acid LB films because of the amorphous nature of polymeric LB films. ${ }^{17,18}$ 4) The chemical structure of the LB films was changeable by using suitable new monomers for polyimide synthesis. Therefore, some functional groups can be readily introduced into the polyimide LB films.

This paper deals with the preparation of polyimide LB film possessing TPP unit, and the application of the LB film to modification of the surface of tin dioxide electrode.

\section{EXPERIMENTAL}

\section{Nitro-TPPs}

To a solution of $19.7 \mathrm{~g}(0.13 \mathrm{~mol})$ of $p$-nitrobenzaldehyde and $13.8 \mathrm{~g}(0.13 \mathrm{~mol})$ of benzaldehyde in $975 \mathrm{ml}$ of propionic acid, $17.4 \mathrm{~g}(0.26 \mathrm{~mol})$ of pyrrole were added dropwise with vigorous stirring at room temperature. The solution was refluxed for $3 \mathrm{~h}$, and the propionic acid was distilled away under reduced pressure. The black colored residue was extracted with chloroform using a Soxhlet extractor. Mononitro-TPP, 5-(4-nitrophenyl)10,15,20-triphenyl-21 $H, 23 H$-porphine, and a mixture of dinitro-TPPs, 5,10-bis(4-nitrophenyl)-15,20-diphenyl-21 $H, 23 H$-porphine, and 5,15-bis(4-nitrophenyl)-10,20-diphenyl$21 H,-23 H$-porphine, were separated by silicagel column chromatography using a mixture of dichloromethane and tetrachloromethane ( $3: 7$ by volume) as eluent. The yields of mononitro-TPP and dinitro-TPPs was $2.88 \mathrm{~g}$ $(5.8 \%)$ and $1.02 \mathrm{~g}(2.2 \%)$, respectively. Besides mono- and di- nitro-TPPs, TPP, trinitro-TPP, and tetranitro-TPP were obtained from the column chromatography.

\section{Diamino-TPPS}

To a solution of $4.13 \mathrm{~g}(5.85 \mathrm{mmol})$ of the mixture of dinitro-TPPs in $500 \mathrm{ml}$ of ethyl acetate, were added $26.4 \mathrm{~g}(0.117 \mathrm{~mol})$ of stannous chloride dissolved in $6 \mathrm{M}$ hydro- chloric acid at room temperature. The mixture was stirred at $80^{\circ} \mathrm{C}$ for $3 \mathrm{~h}$ under nitrogen. The resulting solution was neutralized with a saturated sodium carbonate solution, and extracted with ethyl acetate. cis- and transDiamino-TPPs, 5,10-bis(4-aminophenyl)15,20-diphenyl-21H,23H-porphine (cisDATPP), and 5,15-bis(4-aminophenyl)-10,20diphenyl-21 $H, 23 H$-porphine (trans-DATPP), were separated from the reaction products by silicagel column chromatography using a mixture of dichloromethane and ethyl acetate (19:1 by volume). The yields of cis-DATPP and trans-DATPP were $2.06 \mathrm{~g}(55 \%)$ and $1.40 \mathrm{~g}$ $(37 \%)$, respectively. The IR spectra $(\mathrm{KBr})$ of cis-DATPP exhibited absorptions at 3400 , $1670,1580,1510,1000,800 \mathrm{~cm}^{-1}$, and trans-DATPP exhibited those at 3380, 1670, $1560,1510,980,800 \mathrm{~cm}^{-1} .{ }^{1} \mathrm{H}$ NMR spectra measured in dimethyl sulfoxide- $d_{6}$ of $c i s$ and trans-DATPPs showed peaks as follows ${ }^{19}$; cis-DATPP ( $\delta$ ppm): $8.95(\mathrm{~d}, 4 \mathrm{H}), 8.75(\mathrm{~d}, 4 \mathrm{H})$, $8.35-8.10(\mathrm{~m}, 4 \mathrm{H}), 8.00-7.65(\mathrm{~m}, 10 \mathrm{H}), 7.23$ $(\mathrm{s}, 2 \mathrm{H}), 7.15-6.85(\mathrm{~m}, 4 \mathrm{H}), 5.70(\mathrm{~s}, 4 \mathrm{H})$, trans-DATPP $(\delta \mathrm{ppm}): 9.05(\mathrm{~d}, 4 \mathrm{H}), 8.85(\mathrm{~d}$, $4 \mathrm{H}), 8.40-8.15(\mathrm{~m}, 4 \mathrm{H}), 8.10-7.70(\mathrm{~m}, 10 \mathrm{H})$, $7.30(\mathrm{~s}, 2 \mathrm{H}), 7.20-6.90(\mathrm{~m}, 4 \mathrm{H}), 5.70(\mathrm{~s}, 4 \mathrm{H})$. The UV-visible absorption spectrum ${ }^{19}$ of cis-DATPP measured in chloroform showed peaks at $420(\mathrm{~s}), 518(\mathrm{w}), 554(\mathrm{w}), 595(\mathrm{w})$, and 655 (w) nm. The spectrum ${ }^{19}$ of trans-DATPP exhibited peaks at $418(\mathrm{~s}), 516(\mathrm{w}), 550(2), 590$ (w), and $652(\mathrm{w}) \mathrm{nm}$.

\section{Monoamino-TPP}

Monoamino-TPP (MATPP) was synthesized from mononitro-TPP by the similar reduction procedure to DATPP's synthesis in a yield of $85 \%$. The UV-visible absorption spectrum measured in chloroform showed peaks at $436(\mathrm{~s}), 521(\mathrm{w}), 556(\mathrm{w}), 597(\mathrm{w}), 652$ (w) nm. ${ }^{19}$

\section{Polyamic Acid Containing trans-TPP Unit}

To a solution of $0.598 \mathrm{~g}(0.927 \mathrm{mmol})$ of trans-DATPP in $5 \mathrm{ml}$ of $N, N$-dimethylaceta- 
mide (DMAc), $0.202 \mathrm{~g} \quad(0.927 \mathrm{mmol})$ of pyromellitic dianhydride (PMDA) was added at once. The solution was stirred at $15^{\circ} \mathrm{C}$ for $1 \mathrm{~h}$ and then at $25^{\circ} \mathrm{C}$ for $5 \mathrm{~h}$. The polyamic acid was precipitated by pouring the solution into $300 \mathrm{ml}$ of methanol, and dried under reduced pressure at room temperature. The yield of the polymer was $0.784 \mathrm{~g}(98 \%)$. The inherent viscosity measured at a concentration of $0.5 \mathrm{~g} \mathrm{dl}^{-1}$ in DMAc at $30^{\circ} \mathrm{C}$ was $0.45 \mathrm{dl} \mathrm{g}^{-1}$. The IR spectrum showed characteristic absorptions at 1715,1655 , and $1590 \mathrm{~cm}^{-1}$.

\section{Polyimide LB Film}

Polyimide LB film which possessed TPP unit was prepared using the reported procedure. $^{13-15}$

A solution of the polyamic acid in a mixture of DMAc and benzene ( $1: 1$ by volume) with a concentration of $1 \mathrm{mmol}^{-1}$ was combined with a solution of $N, N$-dimethyloctadecylamine in the same mixed solvent with the same concentration to produce a solution of the polyamic acid amine salt for spread. The measurement of the surface pressure-area isotherm ( $\pi-\mathrm{A}$ curve) was carried out on a San-Esu FSD-20 trough $(500 \mathrm{~mm}$ length and $150 \mathrm{~mm}$ width) with a compression speed of $0.32 \mathrm{~mm} \mathrm{~s}^{-1}$ at $20^{\circ} \mathrm{C}$. The subphase used in the present work was a distilled deionized water. The deposition of $\mathrm{L}$ film of the polyamic acid amine salt was carried out at a surface pressure of 25 and $>5 \mathrm{mN} \mathrm{m}^{-1}$ for upward and downward trip, respectively, onto an appropriate plate (tin dioxide electrode, quartz and glass plates) by dipping through the air-water interface at a rate of $10 \mathrm{~mm} \mathrm{~min}^{-1}$ at $20^{\circ} \mathrm{C}$. The LB film should have the Z-type structure by this deposition procedure.

The conversion of the precursor LB film, thus obtained, to the polyimide LB film was carried out by immersing the LB film in a mixture of acetic anhydride, pyridine, and benzene $\left(1: 1: 3\right.$ by volume) at $20^{\circ} \mathrm{C}$ for $8 \mathrm{~h}$.

\section{$L B$ Film of MATPP}

A solution of MATPP in a $1: 1$ mixture of DMAc and benzene with a concentration of $1 \mathrm{mmol} 1^{-1}$ was added to a solution of stearic acid in the same solvent with the same concentration. The solution was then spread on a deionized water. Measurement of $\pi-A$ curves was carried out by the same procedure as those for the polyamic acid amine salt LB film. The $\mathrm{L}$ film was transferred onto a substrate through the Y-type deposition at a surface pressure of $25 \mathrm{mN} \mathrm{min}^{-1}$.

\section{Measurement of Tilting Angle of Porphyrin Unit $^{20}$}

Ten layers of the LB films were deposited on one side of a glass plate. The tilting angles of porphyrin unit from the plane of the substrate were estimated by using the dichroic ratios obtained from polarized visible spectra using eq 1 , where $D_{0}$ and $D_{45}$ are the dichroic ratios in which the LB films faced at $90^{\circ}$ and $45^{\circ}$ against the incident direction, respectively. The value of $38^{\circ}$ was employed as $\beta$ in this case. The measurements were carried out using the wavelength of $\lambda_{\max }$ of each LB film.

$$
\cos ^{2} \theta=\frac{\left(1 / D_{0}\right)-\left\{1+\left(1 / D_{0}\right) \sin ^{2} \beta\right\} \cdot\left(1 / D_{45}\right)}{\left(1-2 \sin ^{2} \beta\right) \cdot\left(1 / D_{45}\right)-\left\{1+\left(1 / D_{45}\right) \sin ^{2} \beta\right\} \cdot\left(1 / D_{0}\right)}
$$

\section{Measurement of Photocurrent}

The photoelectrochemical properties of the electrode were investigated under nitrogen by using the electrochemical cells, where the tin dioxide electrode, a gold plate, and a saturated calomel electrode (SCE) were used as the working, counter, and reference electrodes, respectively. The LB films on the electrode were kept in contact with an aqueous electrolyte solution of $\mathrm{pH} 4$ containing $10 \mathrm{mM}$ hydroquinone as reducing reagent. Either monochromatic light of $430 \mathrm{~nm}$ or direct xenon 
light without spectroscopic treatment was illuminated to measure the photocurrent.

\section{Measurement Systems}

The IR spectra and UV-visible spectra were measured with a JEOL FT/IR-5000 spectrometer, and a Hitachi U-3400 spectrometer, respectively. The apparatus for the ellipsometry measurements was a NIIC model El-101. Xenon lamp and monochrometer used for the irradiation of light were Ushio VB-501AA $(500 \mathrm{~W})$ and Shimadzu Baush \& Lomb Monochrometer, respectively.

\section{RESULTS AND DISCUSSION}

Monoamino- and trans-diamino-TPPs
(MATPP and trans-DATPP) were synthesized by the reduction of the corresponding nitro compounds, ${ }^{19}$ which were prepared by the method reported by Rothermund ${ }^{21}$ starting from pyrrole, benzaldehyde, and p-nitrobenzaldehyde as shown in eq 2 . The desired nitro compounds, mononitro-TPP 1 and dinitro-TPPs 2 and 3, were separated by the silicagel column chromatography from the reaction mixture including five kinds of tetraphenylporphyrins. Although cis and trans isomers of dinitro compounds, 2 and $\mathbf{3}$, could not be separated in this stage, each pure diamine isomers 5 and $\mathbf{6}$ were obtained by the silicagel column chromatography after the reduction of the nitro group to the amine one.

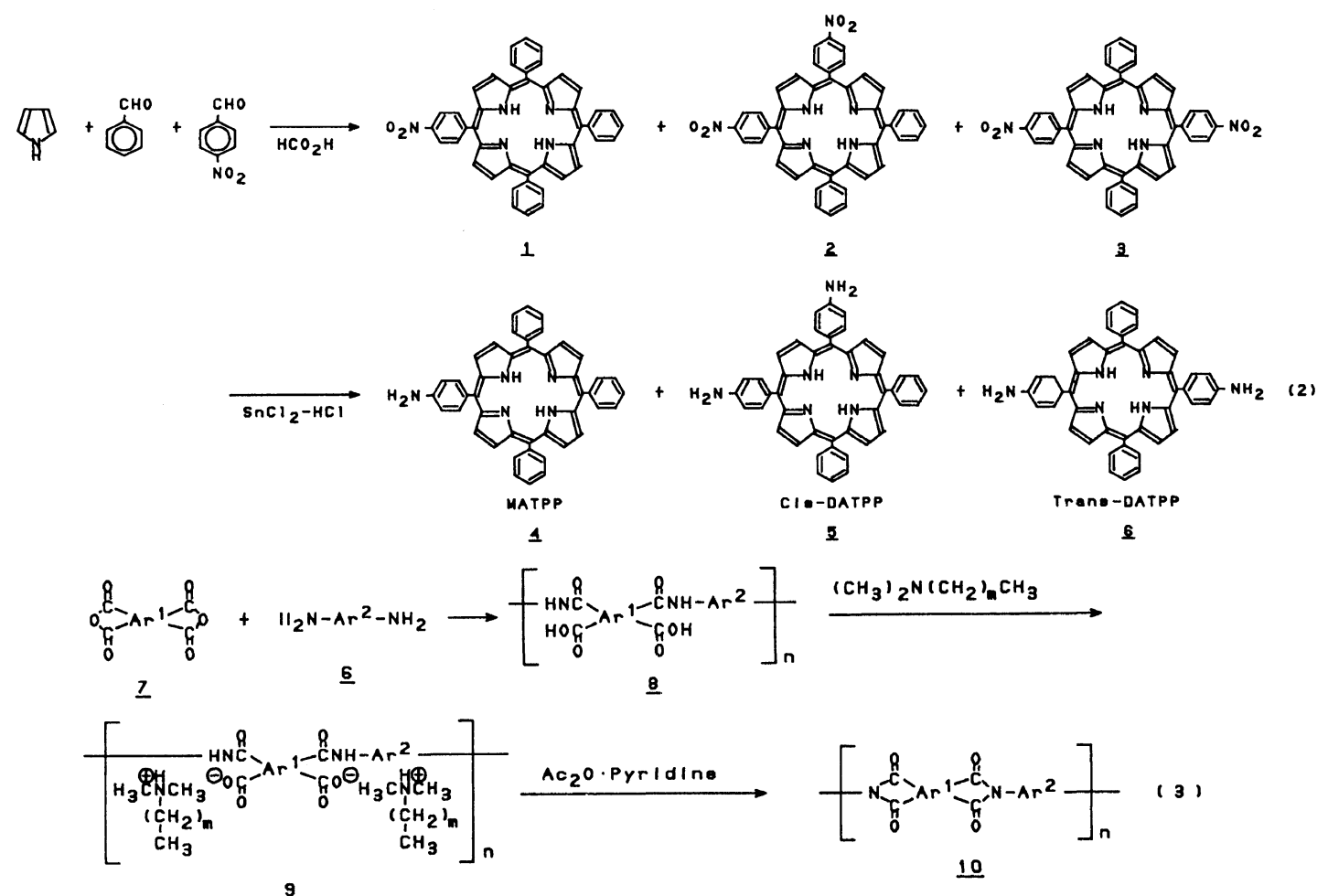

$\underline{9}$

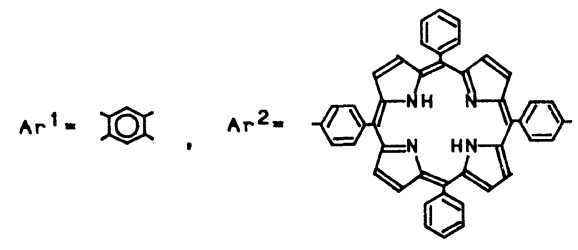


Polyamic acid possessing TPP unit 8 was obtained by the reaction between transDATPP 6 and PMDA 7 with the inherent viscosity of $0.45 \mathrm{dl} \mathrm{g}^{-1}$ measured in DMAc as shown in eq 3 . The LB film of polyimide $\mathbf{1 0}$ containing TPP unit was prepared on an appropriate plate by the procedure described in the previous papers. ${ }^{13-15}$ This procedure consists of three steps as follows: 1) preparation of monolayer film of polyamic acid amine salt having long alkyl chain 9 at an air-water interface, 2) deposition of the L film of 9 onto a substrate, and 3) treatment of the precursor LB film with a mixture of acetic anhydride and pyridine giving the LB film of polyimide $\mathbf{1 0}$. The $\pi-A$ curve of 9 shown in Figure 1 has almost the same rising curve as those of other polyamic acid amine salts, while it shifts to the right hand side because of the bulkiness of TPP unit. ${ }^{13,16}$ Figure 2 shows the relationship of the number of layers and thickness of polyimide LB film measured by the ellipsometry. The linear relationship between these variables indicated that both steps, deposition of the $\mathrm{L}$ film onto the substrate and imidization of the precursor LB film to the polyimide LB film, proceeded smoothly without any problem. The monolayer thickness of the LB film of $\mathbf{1 0}$ was calculated to be $0.6 \mathrm{~nm}$ from the plots of Figure 2.

The tilting angle of TPP unit of the precursor LB film and the polyimide LB film from the plane of the substrate was measured using the dichroic ratio obtained from the polarized visible spectra $(428 \mathrm{~nm}$ for 9 and $438 \mathrm{~nm}$ for 10), where ten layers of each LB film were deposited on one side of a quartz plate. The results of the measurements changing the surface pressure of deposition are listed in Table I. Although the tilting angle increased with increasing the surface pressure, the compression effect was not so remarkable. The tilting angle of TPP unit in the final polyimide LB film was less than $30^{\circ}$.

The $\pi$-A curve of monomeric TPP LB film, consisted of $1: 1$ molar ratio of MATPP and

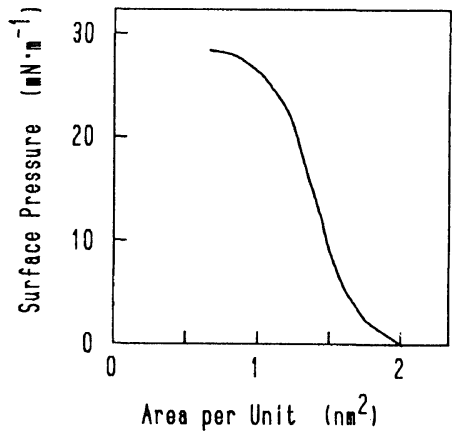

Figure 1. $\pi$-A curve of precursor polymer 9.

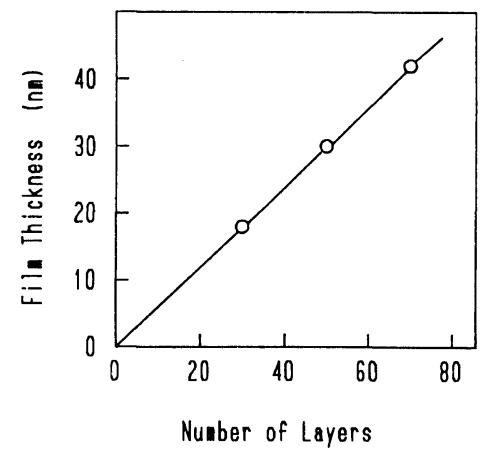

Figure 2. Relationship of thickness and number of layers in LB film of polyimide $\mathbf{1 0}$.

Table I. Relationship between tilting angle of porphyrin unit of LB films and deposition surface pressure

\begin{tabular}{cccc}
\hline \multirow{2}{*}{$\begin{array}{c}\text { Surface } \\
\text { pressure }\end{array}$} & & \multicolumn{2}{c}{ Tilting angle $\left(^{\circ}\right)$} \\
\cline { 1 - 1 } $\mathrm{mN} \mathrm{m}^{-1}$ & & Precursor & Polyimide \\
\cline { 1 - 1 } 15 & & 33.5 & 23.7 \\
20 & & 39.6 & 23.9 \\
25 & 42.1 & 28.6 \\
\hline
\end{tabular}

stearic acid, is shown in Figure 3. The curve possessed a plateau region, and the Y-type deposition was carried out above the region at $25 \mathrm{mN} \mathrm{m}^{-1}$. The tilting angle of the TPP unit was observed to be $38^{\circ}$ in the ten-layers LB film of the mixed MATPP.

Two kinds of semiconductor electrodes, Electrode I modified with LB film of $\mathbf{1 0}$ and Electrode II possessing LB film of the mixture 


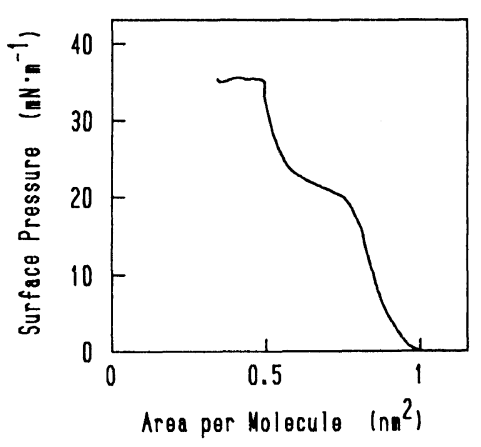

Figure 3. $\pi-\mathrm{A}$ curve of mixture of MATPP and stearic acid $(1: 1)$.

of MATPP and stearic acid, were prepared by deposition of the corresponding LB films onto tin dioxide electrode. The remarkable difference between these two electrodes was absence and presence of the long alkyl spacer chain. The photocurrent measurement was carried out by the wet system shown in Figure 4. This system works as follows: 1) TPP in the LB films is excited by the irradiation of light, 2) the excited electrons are transferred to the tin dioxide electrode, 3) the lacked electrons in TPP are supplied from hydroquinone in the electrolyte solution, and 4) hydroquinone is reproduced at the surface of the counter electrode. The energy diagram of this system is depicted in Figure 5, where the redox potentials are taken note of the previous data. $^{7}$

Figure 6 shows the absorption and photocurrent action spectra, where Electrode I possessed one layer of TPP LB film of $\mathbf{1 0}$. The fact that these two spectra were very similar in shape to each other implied that the electrons were transferred only from the excited TPP to the tin dioxide electrode by the irradiation of light. The blue shift of the maximum peak of the photocurrent spectrum compared with that of the absorption spectrum could be explained by the effect of hydroquinone in the electrolyte solution. The same phenomenon was observed in the absorption spectrum of the LB film after

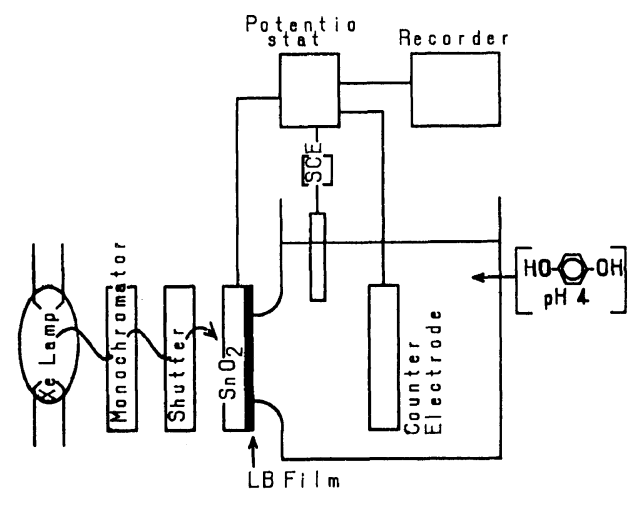

Figure 4. Photocurrent measurement system.

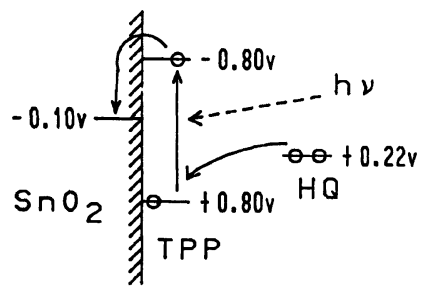

Figure 5. Energy diagram of the electron transfer.

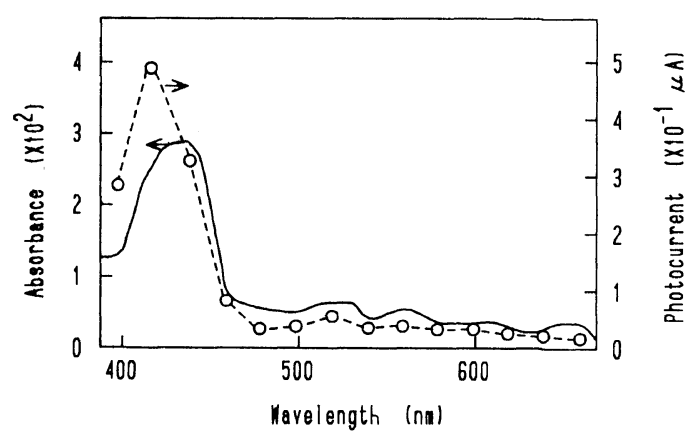

Figure 6. Absorption (solid line) and photocurrent (dotted line) spectra.

immersion in the electrolyte solution.

$$
\eta^{0}=\frac{I_{\mathrm{e}}}{I_{\mathrm{v}}}
$$

The efficiency of transformation of the energy from light to electricity is expressed by the quantum efficiency $\eta^{0}$ given as eq 4 , where $I_{\text {a }}$ is the number of flowing photoelectrons and $I_{\mathrm{v}}$ is the number of photons absorbed by TPP unit. The quantum efficiency of Electrode I with 
Table II. Dependence of number of layers on quantum efficiency $\eta^{0}$

\begin{tabular}{cc}
\hline Number of layers & $\eta^{0}$ \\
\hline 1 & 0.178 \\
5 & 0.092 \\
10 & 0.061 \\
\hline
\end{tabular}

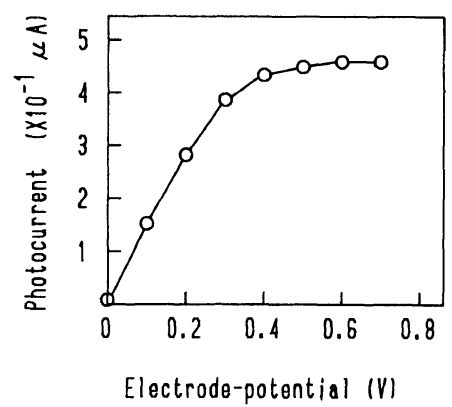

Figure 7. Relationship of photocurrent and electrode potential.

one layer of polyimide LB film was 0.178 . The effect of the number of the layers of the LB films was examined as shown in Table II. The results that the electrode modified with only one layer of the LB film afforded the highest $\eta^{0}$ value meant that the electrons more readily transferred from the closer TPP unit to the tin dioxide electrode surface. Figure 7 shows the electrode-potential dependence of the photocurrent observed under the constant illumination by monochromatic light of $430 \mathrm{~nm}$. The anodic photocurrent increased with increasing of the positive electode potential vs. SCE, and the photocurrent was saturated over $0.4 \mathrm{~V}$.

Next, both Electrode I and Electrode II possessing one layer of the LB films were continuoulsy irradiated to examine the stability of the photocurrent generation. The changes of the photocurrents as a function of irradiation time are shown in Figures 8 and 9, where the monochromatic light of $430 \mathrm{~nm}$, and the whole range of the light coming directly from the xenon lamp without spectroscopic treatment were irradiated, respectively, under applying potential of $0.3 \mathrm{~V}$. Although there

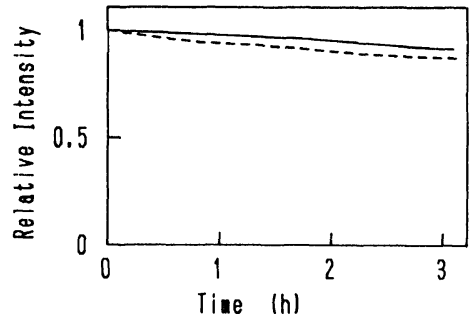

Figure 8. Plots of relative intensity of the photocurrents $v s$. irradiation period of monochromatic light of $430 \mathrm{~nm}$. (solid line, polyimide LB film; dotted line, monomeric LB film.)

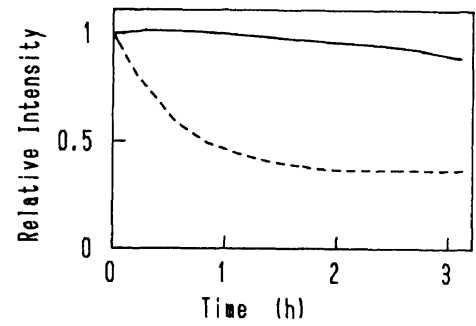

Figure 9. Plots of relative intensity of the photocurrents $v s$. irradiation period of direct xenon light. (solid line, polyimide LB film; dotted line, monomeric LB film.)

was no remarkable change in photocurrent of both electrodes under the irradiation of the monochromatic light, the photocurrent of Electrode II decreased to $50 \%$ of the initial photocurrent level within one hour by the direct irradiation of light of the xenon lamp, and after that, the photocurrent level was kept for a long period. It was remarkable that the photocurrent intensity of Electrode I did not change even after $3 \mathrm{~h}$ under the same irradiation conditions. Thus, the electrons could be transferred more rapidly in Electrode I compared with Electrode II especially under the stronger irradiation condition. The LB films did not come off from the surface of the tin dioxide electrode during the operation of the electron trnasfer reaction even in the case of Electrode II, judging from the fact that the absorption spectra of both electrodes did not change before and after the direct irradiation of the light. The long term stability of the photocurrent in Electrode I can be explained 
by the hydrophobicity of the surface of the electrodes and absence of the long alkyl chain. Since refilling of the lacked electrons in the excited TPP unit by the reducing agent is extremely necessary in the present reaction system as shown in Fig. 5, the contact of TPP unit with hydroquinone should decide the level of the photocurrent. TPP unit in the Electrode I could directly contact with hydroquinone molecule in the electrolyte, while the contact was interfered by the hydrophobic long alkyl chain in the case of Electrode II. The photocurrent intensity of Electrode II decreased with the irradiation, and reached to an equilibrium under the stronger irradiation. This phenomenum can be explained as follows; the rate of refilling of the lacked electrons in TPP was slower than that of the transfer of the electrons from the excited TPP to the tin dioxide electrode.

The hydrophobicity of the surface of the electrode could be estimated by the contact angle of water on the one-layer LB film deposited on a glass plate, which were measured as $28^{\circ}$ and $77^{\circ}$ for the LB films corresponding to Electrode I and II, respectively. On the other hand, the fact that Electrode II showed the almost the same level of the photocurrent of Electrode I under the lower energy of irradiation with the spectroscopic treatment indicated that the refilling rate of lacked electron of TPP unit from hydroquinone should be comparable to that of Electrode I.

In conclusion, the tin dioxide electrode was successfully modified by polyimide LB film containing TPP unit. This electrode showed a higher stability compared with the electrode modified by the monomeric TPP LB film against the continuous irradiation of Xenon light without the spectroscopic treatment. Thus, the polyimide LB film which possesses no long alkyl spacer chains exhibits and advantage in the electron transfer reactions.

\section{REFERENCES}

1. A. L. Lehninger, "Biochemistry," 2nd ed, Worth, New York, 1975.

2. N. N. Lichtin, Chemtech., 10, 254 (1980).

3. T. Katsu, K. Tamagake, and Y. Fujita, Chem. Lett., 289 (1980)

4. P. A. Breddels and G. Blasse, J. Chem. Soc., Fraday Trans. 2, 80, 1055 (1984).

5. M. Matsumura, K. Mitsuda, N. Yoshizawa, and H. Tsubomura, Bull. Chem. Soc. Jpn., 54, 692 (1981).

6. H. TiTien and J. Higgins, Chem. Phys. Lett., 93, 276 (1982).

7. M. Fujihira, T. Kubota, and T. Osa, J. Electroanal. Chem., 119, 379 (1981).

8. For special issue of LB films: Thin Solid Films, 68 (1980), 99 (1983), and 132, 133, and 134 (1985), and 159 and 160 (1988), 178, 179, and 180 (1989).

9. M. Fujihira, Oyo Butsuri, 57, 1890 (1988).

10. T. Miyasaka, T. Watanabe, A. Fujishima, and K. Honda, J. Am. Chem. Soc., 100, 6657 (1978).

11. T. Miyasaka, T. Watanabe, A. Fujishima, and K. Honda, Nature, 277, 638 (1979).

12. T. Miyasaka, T. Watanabe, A. Fujishima, and K. Honda, Photochem. Photobiol., 22, 217 (1980).

13. M. Suzuki, M. Kakimoto, T. Konishi, Y. Imai, M. Iwamoto, and T. Hino, Chem. Lett., 395 (1986).

14. M. Kakimoto, M. Suzuki, T. Konishi, Y. Imai, M. Iwamoto, and T. Hino, Chem. Lett., 823 (1986).

15. M. Kakimoto, M. Suzuki, Y. Imai, M. Iwamoto, and T. Hino, "Polymers for High Technology, Electronics and Photonics," M. J. Bowden and S. R. Turner, Eds., ACS Symposium Series 346, American Chemical Society, Washington, DC, 1987, p 484.

16. Y. Nishikata, T. Konishi, A. Morikawa, M. Kakimoto, and Y. Imai, Polym. J., 20, 269 (1988).

17. Y. Nishikata, M. Kakimoto, A. Morikawa, I. Kobayashi, Y. Imai, Y. Hirata, K. Nishiyama, and M. Fujihira, Chem. Lett., 861 (1989).

18. H. Matsuda, K. Sakai, H. Kawada, K. Eguchi, and T. Nakagiri, J. Mol. Electron., 5, 107 (1989).

19. H. Xie, S. Han, K. Cui, Y. Liu, and C. Zhang, Gaodeng Xuexiao Huaxue Xuebao, 9, 859 (1988) [Chem. Abstr., 111, 7124n (1989)].

20. M. Yoneyama, M. Sugi, M. Saito, K. Ikegami, S. Kuroda, and S. Iijima, Jpn. J. Appl. Phys., 25, 961 (1986).

21. P. Rothemund, J. Am. Chem. Soc., 57, 2010 (1935). 